\title{
Consensus Virtual Output Impedance Control based on the Novel Droop Equivalent Impedance Concept for a Multi-bus Radial Microgrid
}

\author{
Yi Chyn Cassandra Wong, Student Member, IEEE, Chee Shen Lim, Senior Member, IEEE, \\ Mihai Dragos Rotaru, Member, IEEE, Andrew Cruden, Xin Kong
}

\begin{abstract}
Most of the existing reactive power sharing schemes that assume parallel architecture are known to be less effective for multi-bus radial microgrids. This paper proposes an improved reactive power sharing scheme that exploits the novel concept of droop equivalent impedance into designing a consensus virtual-output-impedance-based droop control scheme. The control scheme leads to two notable improvements: (a) it proves that only either virtual resistance or virtual reactance is sufficient to restore proportionate reactive power sharing; (b) only a global coupling gain needs to be tuned and no proportional-integral controller is required. A systematic guideline that establishes the approximate range of stable coupling gain is developed. This simplifies the tuning process of the coupling gain. The power correction performance, the resulting bus voltage behavior, consensus control stability, and the robustness to time delay have been investigated in conjunction with an islanded microgrid modified from the IEEE 34 Node Test Feeder. It is shown that the consensus control scheme is capable to demonstrate accurate power sharing regardless of the changes in the network topology, network impedances, loading conditions, and communication delay.
\end{abstract}

Index Terms - Droop control, radial microgrid, reactive power sharing, adaptive virtual output impedance.

\section{INTRODUCTION}

$\mathrm{I}_{\mathrm{s}}^{\mathrm{n}}$ $\mathrm{n}$ order to facilitate the requirement of autonomous power sharing among distributed energy resources (DERs) in a microgrid, droop control mechanism has been widely adopted. However, it is known that although the load active power can be proportionately shared among the DERs, the load reactive power tends to suffer from the problem of non-proportional sharing. This is due fundamentally to non-negligible voltage

Manuscript received Sep. 11, 2019; revised Dec. 10, 2019. Accepted Jan 28, 2020. This work was supported by the Fundamental Research Grant Scheme (FRGS/1/2016/TK07/USMC/02/1) awarded by the Ministry of Higher Education, Malaysia. (Corresponding author: Chee Shen Lim)

Cassandra Y. C. Wong and C. S. Lim are with University of Southampton Malaysia, 79200 Iskandar Puteri, Malaysia (e-mail: y.c.c.wong@soton.ac.uk, c.s.lim@soton.ac.uk).

M. D. Rotaru is with the Institute of Microelectronics, A*STAR, 627590 Singapore (e-mail: Mihai_Dragos_Rotaru@ime.a-star.edu.sg).

A. Cruden is the Energy Technologies Research Group, University of Southampton, Southampton SO17 1BJ, U.K. (e-mail: a.j.cruden@soton.ac.uk)

X. Kong is with the Experimental Power Grid Centre of ERI@N, Nanyang Technological University, 627590 Singapore (e-mail: kong_xin@ntu.edu.sg). discrepancies at the points of coupling [1]-[3].

Various reactive power sharing improvement techniques have been developed in the past and, in the authors' opinion, they can be broadly categorized to communication-less and communication-based techniques. The former is typically developed for parallel DERs (i.e. those share a point of common coupling through respective feeder impedances) and is predominantly based on the fundamental principle of improving the droop control design. One example being the cross-coupled droop control reported in [4]. Though with higher reliability, they cannot be directly extended to multi-bus radial microgrid cases, especially the ones with the plug-and-play requirement [1]. The problem has been solved predominantly through the communication-based techniques (with some exception, as will be explained next). It is worth highlighting that multi-bus radial architecture is in practice very relevant for large-area distribution microgrids. Summarily, the improved communication-based reactive power correction techniques are based primarily on two types of correction mechanisms: one based on the adjustment of droop parameters (e.g. droop coefficient or dispatch command); one based on virtual output impedance (VOI) integrated droop control and through adjusting the VOI value. Examples of the former type include the decentralized control based dispatch command adjustment [5], adaptively tuned droop coefficient [6], optimal no-load reference through primal-dual interior-point method [7], a twolayer consensus algorithm with no-load reference adjustment [8], a distributed-averaging droop control with adjustable dispatch [9], and a consensus control with local voltage restoration and reactive power mismatch correction [10]. Differently, a consensus-based power sharing control is proposed in [11] and [12] through output current mismatch. In addition, [13] has introduced a fundamentally-different distributed control scheme that uses the output voltage reference derived directly from the distributed errors instead of following the usual droop mechanism. It was however reported that the system's equilibrium and stability has subsequently become heavily dependent on the choice of control parameters.

VOI based control schemes were first introduced in [14] and [15]. These methods have assumed a parallel architecture and 
therefore only considered the mismatch of output/feeder impedances but not network impedances. A similar fixedvirtual-impedance techniques have been reported in [3] and [16] but only for microgrids with unchanging, i.e. non-plugand-play, parallel architecture (owing to the line impedance parameter requirement). A communication-less offline geneticalgorithm-based VOI scheme for a radial microgrid is proposed in [17]. Similarly, [18] has reported a communication-less particle-swarm-optimization-based droop control with fixedVOI for power sharing improvement. However, their extension to microgrids with changing topology is also not possible. Some other schemes that focus on unbalance and/or harmonic power sharing can be found in [2], [19], [20]. In addition, [21] has shown that the virtual impedance value is critical in gridconnected mode (not within the scope of this work) and they should be designed to be kept within the desired range to prevent instability.

As far as accurate reactive power sharing in an islanded radial microgrid is concerned, [17], [22]-[26] are relevant ([26] only focuses on non-changing network topology). Note that some literature (e.g. [23]) have used the term "mesh" instead of "radial” [27]. The latter seems to be a more usual definition to describe the topology of the network under consideration, and hence is the one being used in what follows. An adaptive VOI control with centralized secondary integrator control is presented in [25]. The work has shown that by equating the virtual resistance to the virtual reactance and by adjusting their values through integral control, the reactive power can be made equal. Another similar centralized scheme is proposed in [22]. This work concludes that reactive power sharing can be corrected by adjusting the virtual resistance alone (through proportional-integral control) but keeping the virtual reactance constant. Another centralized secondary control supplemented adaptive VOI scheme is proposed in [23]. The work achieves the correction by adjusting the virtual reactance (through proportional-integral control) while keeping the ratio of virtual resistance/reactance constant. In the distributed VOI scheme proposed by [24], it is shown that reactive power sharing correction can be realized by adjusting both the virtual resistance and reactance based on the distributed reactive power sharing errors (again, through proportional-integral control). Two practical issues are concluded here: there is no systematic way in determining the ratio of virtual reactance/resistance ratio; tuning of the coupling and proportional-integral gains have not been discussed comprehensively (e.g. [25] highlighted that the integral gain has to be set small to ensure stability; [22][24] reported that, with a fixed static component, the dynamic component is deliberately tuned via a small integral gain).

The work intends to contribute in three aspects. Firstly, this work will visit the problem through the concept of droop equivalent impedance. It will be shown that, as far as reactive power sharing of an islanded radial microgrid is concerned, there is never a need to have both virtual reactance and resistance, but only either of them is sufficient to correct the reactive power sharing. Secondly, the proposed control scheme adopts a single consensus integral action without needing any

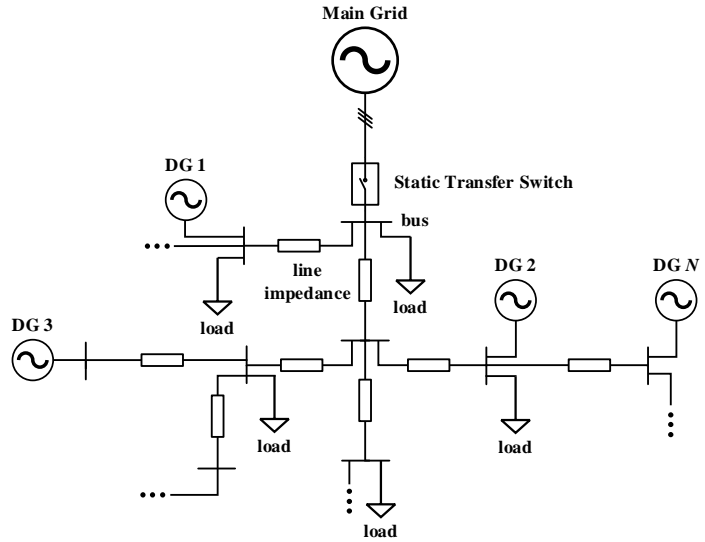

Fig. 1. Architecture of a multi-bus radial microgrid.

proportional-integral controllers. This simplifies the gain tuning process of the distributed system. Thirdly, as mentioned in [9], pure-droop-based power control is non-linear, hence some forms of linearization is required to establish the behavior of the voltage/power towards the coupling gain. The proposed adaptive-VOI-based power control has an additional non-linear behavior due to the adaptive VOI nature. Hence the coupling gain tuning would be a more critical issue. This work establishes a systematic guideline that determines the approximate range of stable coupling gain. In essence, the guideline first linearizes the non-linear system about the probable operating regions, then establishes the range of coupling gain that ensures convergence through the standard linear consensus theorem.

The remaining of this paper is organized as follows. Section II introduces the fundamentals of consensus algorithm. Section III introduces the novel droop equivalent impedance together with other commonly known impedances. Section IV discusses the consensus VOI-based control scheme and the coupling gain tuning guideline. Section $\mathrm{V}$ introduces the islanded microgrid network, the selected primary controls, and the results under three test cases: static and transient load, plug-and-play capability, and communication delay. Section VI concludes the paper.

\section{Preliminaries OF CONSENSUS CONTROL}

The communication network of a microgrid can be modeled as a graph with edges corresponding to the information flow between the DERs, denoted as the nodes. The graph is generally expressed as $\boldsymbol{G}=\left(\boldsymbol{V}_{G}, \boldsymbol{E}_{G}, \boldsymbol{A}_{G}\right)$ with a set of $N$ nodes $\boldsymbol{V}_{G}=\left\{v_{1}, v_{2}\right.$, $\left.\ldots, v_{N}\right\}$, a set of edges $\boldsymbol{E}_{G} \subset \boldsymbol{V}_{G} \times \boldsymbol{V}_{G}$ and an adjacency matrix $\boldsymbol{A}_{G}$ $=\left[a_{i j}\right] \in \mathbb{R}^{N \times N}$. The edges $\boldsymbol{E}_{G}$ denote the communication links between DERs and each edge $\left(v_{j}, v_{i}\right) \in \boldsymbol{E}_{G}$ represents the information flow from node $j$ to node $i$, is associated with a weight $a_{i j}$, with $a_{i j}>0$ if $\left(v_{j}, v_{i}\right) \in \boldsymbol{E}_{G}$, otherwise $a_{i j}=0$. Node $j$ is called a neighbor of node $i$ if $\left(v_{j}, v_{i}\right) \in \boldsymbol{E}_{G}$ and the set of neighbors of node $i$ is denoted as $N_{i}=\left\{v_{j}:\left(v_{j}, v_{i}\right) \in \boldsymbol{E}_{G}\right\}$. The graph is termed to be undirected if $\left(v_{j}, v_{i}\right) \Rightarrow\left(v_{i}, v_{j}\right) \in \boldsymbol{E}_{G}$; otherwise directed. The in-degree matrix is defined as $\boldsymbol{D}=\operatorname{diag}\left\{d_{i}\right\} \in \mathbb{R}^{N \times N}$ with $d_{i}=\sum$ ${ }_{j \in N_{i}} a_{i j}$ and hence, the Laplacian matrix of the graph is defined as $\boldsymbol{L}_{G}=\boldsymbol{D}-\boldsymbol{A}_{G}$. 


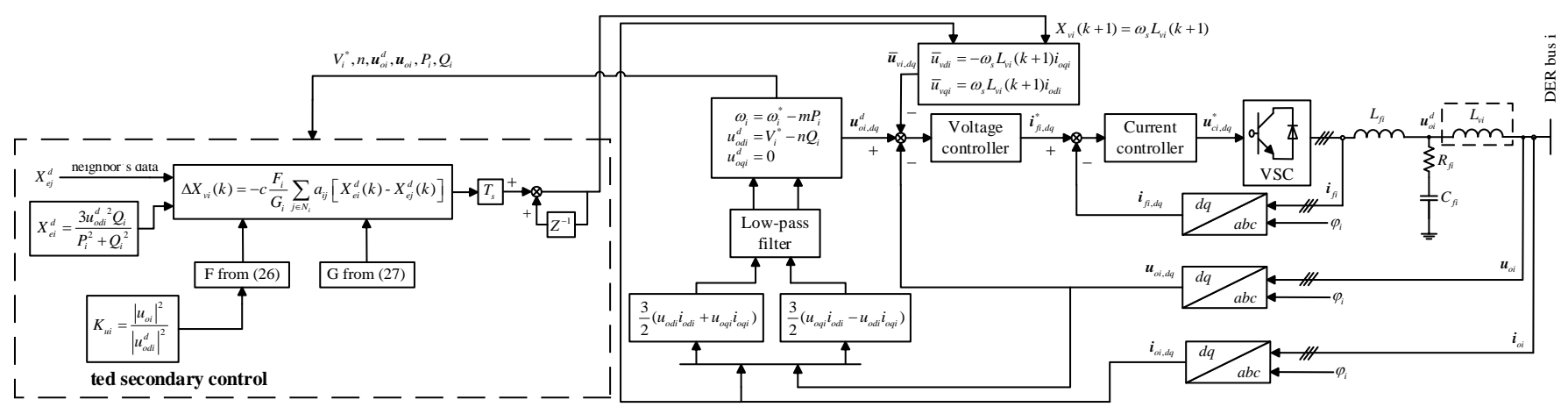

Fig. 2. Schematic diagram of the proposed consensus virtual output impedance control of a droop-based distributed energy resource.

Consider the first-order discrete-time consensus algorithm

$$
\begin{aligned}
& x_{i}(k+1)=x_{i}(k)+\delta_{i}(k) \\
& \delta_{i}(k)=-c \sum_{j \in N_{i}} a_{i j}\left[x_{i}(k)-x_{j}(k)\right]
\end{aligned}
$$

where $x_{i}$ and $\delta_{i}$ denote the state variable and control input. It is established that consensus is guaranteed if the coupling gain $c$ satisfies the sufficient condition [28]

$$
c<\frac{1}{d_{\max }}
$$

where $d_{\max }=\max (\boldsymbol{D})$. Suppose that information is passed between the DERs with a time-delay $\tau>0$, the consensus algorithm can be written as

$$
\begin{aligned}
& x_{i}(k+1)=x_{i}(k)+\delta_{i}(k) \\
& \delta_{i}(k)=-c \sum_{j \in N_{i}} a_{i j}\left[x_{i}(k-\tau)-x_{j}(k-\tau)\right]
\end{aligned}
$$

The time-delayed consensus control is said to be globally asymptotically stable if and only if [24], [29]-[31]

$$
0<\tau<\frac{\pi}{2 \lambda_{\max } c}
$$

where $\lambda_{\max }$ is the maximum eigenvalue of the communication graph's Laplacian matrix $\boldsymbol{L}_{\mathrm{G}}$.

\section{Control Modeling AND AnAlysis OF Multi-Bus RADIAL MICROGRID}

A microgrid is considered radial structured with DERs and/or loads connected to the local distributed network at various nodes. Fig. 1 depicts the configuration/topology of an islanded multi-bus radial microgrid composed of $N$ number of DERs and loads.

\section{A. Primary Control and Virtual Output Impedance Integrated Droop Control}

The primary control of a VOI-controlled DER includes a power control, an output voltage control, an inverter current control and a virtual output impedance control, as shown in Fig. 2. During islanded operation, DERs achieve autonomous power sharing through the standard active power-frequency $(P-f)$ and reactive power-voltage $(Q-V)$ droop control [1], [32], [33], expressed as

$$
\begin{gathered}
\omega_{i}=\omega_{i}^{*}-m P_{i} \\
V_{i}=V_{i}^{*}-n Q_{i}
\end{gathered}
$$

where $\omega_{i}$ and $V_{i}$ are the per-unit operating frequency and the droop voltage amplitude (normalized to network's phase peak

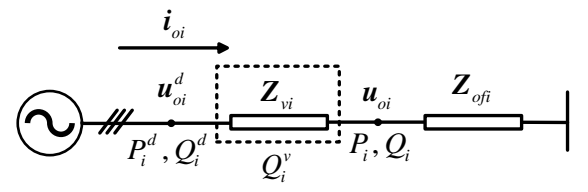

(a)

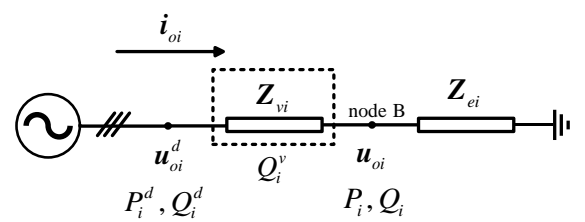

(b)

Fig. 3. (a) Single line diagram for an inverter-based DER with a virtual output impedance $\left(\boldsymbol{Z}_{v i}\right)$ and a feeder impedance $\left(\boldsymbol{Z}_{o f i}\right)$; (b) the corresponding single line diagram with the virtual output impedance and a physical equivalent impedance as seen by the DER output towards the microgrid network, $\boldsymbol{Z}_{e i}$.

voltage) of $i^{\text {th }} \mathrm{DER}, \omega_{i}^{*}$ and $V_{i}^{*}$ are corresponding per-unit noload frequency and voltage reference, and $m$ and $n$ are the perunit droop coefficients. Typically, $m$ and $n$ are commonly shared among the DERs in the same microgrid. $P_{i}$ and $Q_{i}$ are the filtered per-unit active and reactive output powers (normalized to the respective $\mathrm{kVA}$ rating).

Since all the inner-loop controllers operate in the synchronous reference frame, the direct $(d)$ and quadrature $(q)$ notations will be used in what follows. In each DER, the synchronous reference frame is made aligned to the droop output voltage vector $\left(\boldsymbol{u}_{o i}^{d}=u_{o d}^{d}+j u_{\text {oqi }}^{d}, u_{\text {odi }}^{d}\right.$ and $u_{\text {oqi }}^{d}$ being the $d$ and $q$-axis components normalized to the phase peak voltage), the voltage components can be expressed as:

$$
\begin{aligned}
u_{\text {odi }}^{d} & =V_{i} \\
& =V_{i}^{*}-n Q_{i} \\
u_{o q i}^{d} & =0
\end{aligned}
$$

The synchronous reference frame's angular position is obtainable from the respective droop frequency in (5) after synchronizing with the microgrid during first connection.

The active and reactive output powers with low-pass filtering (and with amplitude-invariant rotational transformation) can be written as

$$
\begin{aligned}
& \tau_{c} \frac{d P_{i}}{d t}+P_{i}=\frac{3}{2}\left(u_{o d i} i_{o d i}+u_{o q i} i_{o q i}\right) \\
& \tau_{c} \frac{d Q_{i}}{d t}+Q_{i}=\frac{3}{2}\left(u_{o q i} i_{o d i}-u_{o d i} i_{o q i}\right)
\end{aligned}
$$

where $\tau_{c}$ is the low-pass filter's cutoff period and $u_{\text {odi }}, u_{\text {oqi }}, i_{\text {odi }}$, 
and $i_{o q i}$ are the $d$ - and $q$-axis measured output voltage and current components.

VOI control works by regulating the output voltage references from the standard droop control, given as

$$
\begin{aligned}
& u_{o d i}^{*}=u_{o d i}^{d}-u_{v d i} \\
& u_{o q i}^{*}=-u_{v q i}
\end{aligned}
$$

where $u_{\text {odi }}^{*}$ and $u_{\text {oqi }}^{*}$ are the output voltage references after the VOI, and $u_{v d i}$ and $u_{v q i}$ are the voltage drop components across the VOI. The electrical steady state values (i.e. $\bar{u}_{v d i}$ and $\bar{u}_{v q i}$, after assuming $d / d t=0$ ) are calculated as

$$
\begin{aligned}
& \bar{u}_{v d i}=R_{v i} i_{o d i}-\omega_{s} L_{v i} i_{o q i} \\
& \bar{u}_{v q i}=R_{v i} i_{o q i}+\omega_{s} L_{v i} i_{o d i}
\end{aligned}
$$

where $\omega_{s}$ is the nominal angular frequency in rad/s, and $R_{v i}$ and $L_{v i}$ are the virtual resistance and inductance.

\section{B. Reactive Power Sharing Strategy based on the Droop Equivalent Impedance Concept}

In per-phase quantity, we first introduce the following impedance terms: droop equivalent impedance $\left(\boldsymbol{Z}_{e i}^{d}=R_{e i}^{d}+\right.$ $\left.j X_{e i}^{d}\right)$, physical equivalent output impedance $\left(Z_{e i}=R_{e i}+j X_{e i}\right)$, virtual output impedance $\left(\boldsymbol{Z}_{v i}=R_{v i}+j X_{v i}\right)$, and feeder impedance $\left(\boldsymbol{Z}_{o f}=R_{o f i}+j X_{o f}\right)$ of $i^{\text {th }}$ DER. The latter three can be seen directly from Fig. 3. In Fig. 3a, a commonly seen single-line diagram that consists of the virtual output impedance and the physical feeder impedance is shown. In Fig. 3b, an equivalent single-line diagram that replaces both the feeder impedance and the grid connection with a physical equivalent impedance, is shown. Since the voltage vector at node A is synchronized to the droop angular position (i.e. $u^{d}{ }_{o q i}=0 \mathrm{~V}$ ), the phase voltage amplitude can be obtained directly from the $d$-axis component, which in turn equals to the droop output voltage reference (as reflected in eq. (6)). The concept of droop equivalent impedance is introduced by first defining the following:

$$
\boldsymbol{Z}_{e i}^{d}=\frac{3 u_{o d i}^{d 2}}{\boldsymbol{S}_{i}^{*}}=\frac{3 u_{\text {odi }}^{d 2}}{P_{i}-j Q_{i}}
$$

where $\boldsymbol{S}_{i}\left(=P_{i}+j Q_{i}\right)$ is the fundamental apparent power at node $\mathrm{B}$ and $u_{\text {odi }}^{d}$ is droop output voltage at node A. It is important to highlight that this impedance is different from the commonly known physical equivalent impedance $\boldsymbol{Z}_{e}$, defined below (with $u_{o i}$ being the normalized output voltage after VOI):

$$
\boldsymbol{Z}_{e i}=\frac{3 u_{o i}{ }^{2}}{\boldsymbol{S}_{i}^{*}}=\frac{3 u_{o i}{ }^{2}}{P_{i}-j Q_{i}}
$$

A common design principle of droop control is that all the generating sources should be adopting the same droop profile (although it is acknowledged here that some variations do exist in some microgrid literature). Upon steady state before reactive power correction, since droop frequency is a global uniform variable, the active power can always be accurately (i.e. proportionately) shared among DERs [1], [9], [23], [25]. This gives (with $t$ represents time):

$$
\lim _{t \rightarrow \infty}\left[P_{1}(t)=P_{2}(t)=\ldots=P_{N}(t)\right]
$$

However, owing to potentially significant voltage discrepancies at their points of coupling in a multi-bus radial microgrid, the reactive power, if left uncompensated, will not be proportionately shared among the DERs. Upon success correction by the VOI-based control schemes, one can expect that

(i) the reactive power will be proportionally shared:

$$
\lim _{t \rightarrow \infty}\left[Q_{1}(t)=Q_{2}(t)=\ldots=Q_{N}(t)\right]
$$

(ii) the droop output voltage reference for all DERs will have a common value:

$$
\lim _{t \rightarrow \infty}\left[u_{o d 1}^{d}(t)=u_{o d 2}^{d}(t)=\ldots=u_{o d N}^{d}(t)\right]
$$

With conditions (12-14), it can be realised from (10) that the droop equivalent impedances of all DERs must also converge to a common value:

$$
\lim _{t \rightarrow \infty}\left[Z_{e 1}^{d}(t)=Z_{e 2}^{d}(t)=\ldots=Z_{e N}^{d}(t)\right]
$$

Some previous works [6], [16], [26], [31] have exploited the concept of converging the DERs' equivalent impedance of $\left(\boldsymbol{Z}_{v i}\right.$ $+\boldsymbol{Z}_{\text {of }}$ ) (see Fig. 3a) to a common value to realise proportional reactive power sharing. However, since the concept requires a common point of coupling, it can be deduced that the method is not applicable to a multi-bus radial microgrid. The work intends to introduce a novel concept to address this limitation, as explained next. One useful observation of (15) is that if the virtual output impedance $\boldsymbol{Z}_{v i}$ can be controlled in such a way that the droop equivalent impedance $\boldsymbol{Z}^{d}{ }_{e i}$ equalizes, then the reactive power will be shared proportionately. On this basis, we propose that the following consensus protocol:

$$
\begin{aligned}
& \dot{\boldsymbol{Z}}_{e i}^{d}=\boldsymbol{\delta}_{i} \\
& \boldsymbol{\delta}_{i}=-c_{i} \sum_{j \in N_{i}} a_{i j}\left(\boldsymbol{Z}_{e i}^{d}-\boldsymbol{Z}_{e j}^{d}\right)
\end{aligned}
$$

Based on (16), it can be deduced that one can drive $\mathbf{Z}^{d}{ }^{d}$ towards a consensus value, then the proportional reactive power sharing will be achieved. Nevertheless, it was found that (16) can be simplified further. This is explained by first expanding (10):

$$
R_{e i}^{d}+j X_{e i}^{d}=\left(\frac{3 u_{\text {odi }}^{d 2} P_{i}}{P_{i}^{2}+Q_{i}^{2}}\right)+j\left(\frac{3 u_{o d i}^{d}{ }^{2} Q_{i}}{P_{i}^{2}+Q_{i}^{2}}\right)
$$

Based on (12), (14) and (17), it can be deduced that:

(i) if the droop equivalent reactance $\left(X_{e}^{d}\right)$ of all DERs were to converge to a common value, a proportional reactive power sharing will be achieved. With these conditions, the droop equivalent resistance $\left(R_{e}^{d}\right)$ will also equalize;

(ii) if the droop equivalent resistance $\left(R_{e}^{d}\right)$ of all DERs were to converge to a common value, a proportional reactive power sharing will be achieved. With these conditions, the droop equivalent reactance $\left(X^{d}{ }_{e}\right)$ will also equalize.

In essence, this means that as far as reactive power correction is concerned, there is seldom a need to regulate both droop equivalent impedance components. In fact, any attempt to regulate both together would be an ill-posed problem and will result in control instability. This observation somewhat agrees with most previous works, e.g. changing $R_{v}$ fixed $X_{v}$ [17], [22], [34]; fixed $R_{v} / X_{v}$ ratio [23]-[25]. In [24], although there are individual virtual resistive and reactance control loops, however, the two virtual impedance components are inherently coupled since their integral loop is common (which leads to an approximately fixed dynamic $R_{v} / X_{v}$ ratio). Therefore, moving forward, it is proposed here that only the droop equivalent reactance is considered, i.e.:

$$
\dot{X}_{e i}^{d}=-c \sum_{j \in N_{i}} a_{i j}\left(X_{e i}^{d}-X_{e j}^{d}\right)
$$


Note that one may also use the droop equivalent resistance as the consensus error, but it can be seen from (17) that the droop equivalent reactance has a more direct impact on the control of reactive power and hence is the preferred choice.

\section{AdAPTIVE ViRTUAL OUTPUT IMPEDANCE CONTROL BASED ON DROOP EQUIVALENT IMPEDANCE CONSENSUS}

\section{A. Proposed Consensus-VOI Control based on Droop Equivalent Impedance}

Most of the existing consensus-based reactive power correction schemes are based on the reactive power values, e.g. $Q_{i}$ as in [5], [8], [13]; and $n_{i} Q_{i}$ [10], [24], [31]. Based on the deduction in Section III, it is therefore proposed here that the consensus protocol is applied to the droop equivalent reactance $X^{d}{ }_{e i}$. However, it can be easily confirmed that (18) is still not suitable for implementation as there is no direct mean to control $X^{d}{ }_{e i}$ but only $X_{v i}$ is directly controllable. Hence, their relationship is first analyzed in what follows.

One can define the reactive power injected by a DER (from node A) in terms of the virtual output impedance and the physical equivalent impedance (as shown in Fig. 3b):

$$
Q_{i}^{d}=\frac{3 u_{o d i}^{d{ }^{2}}\left(X_{e i}+X_{v i}\right)}{R_{e i}^{2}+\left(X_{e i}+X_{v i}\right)^{2}}
$$

where the physical equivalent resistance $R_{e i}$ and reactance $X_{e i}$ can be expanded from (11) to become

$$
R_{e i}=\frac{3 u_{o i}{ }^{2} P_{i}}{P_{i}^{2}+Q_{i}{ }^{2}} \text { and } X_{e i}=\frac{3 u_{o i}{ }^{2} Q_{i}}{P_{i}^{2}+Q_{i}{ }^{2}}
$$

Then, the virtual reactive power across $X_{v i}$ can be written as

$$
Q_{i}^{v}=\frac{3 i_{o i}^{2} X_{v i}^{2}}{X_{v i}}
$$

where the normalized output current $i_{o i}$ can be expressed in terms of $u_{\text {odi }}^{d}$ and the physical equivalent impedance as:

$$
i_{o i}^{2}=\frac{u_{o d i}^{d 2}}{R_{e i}^{2}+\left(X_{e i}+X_{v i}\right)^{2}}
$$

Then, a relationship between $X_{v i}$ and the reactive output power $Q_{i}$ can be expressed as

$$
\begin{aligned}
Q_{i} & =Q_{i}^{d}-Q_{i}^{v}=\frac{3 u_{o d i}^{d} X_{e i}}{R_{e i}^{2}+\left(X_{e i}+X_{v i}\right)^{2}} \\
X_{v i} & =\sqrt{\frac{3 u_{o d i}^{d^{2}} X_{e i}}{Q_{i}}-R_{e i}^{2}}-X_{e i}
\end{aligned}
$$

Substitute (6) and (20) into (23) results in

$$
\begin{aligned}
X_{v i} & =\sqrt{\frac{3\left(V_{i}^{*}-n Q_{i}\right)^{2} K_{u i} \frac{3\left(V_{i}^{*}-n Q_{i}\right)^{2} Q_{i}}{P_{i}^{2}+Q_{i}^{2}}}{Q_{i}}-\left(K_{u i} \frac{3\left(V_{i}^{*}-n Q_{i}\right)^{2} P_{i}}{P_{i}^{2}+Q_{i}^{2}}\right)^{2}} \\
& -K_{u i} \frac{3\left(V_{i}^{*}-n Q_{i}\right)^{2} Q_{i}}{P_{i}^{2}+Q_{i}^{2}}
\end{aligned}
$$

\begin{tabular}{|c|c|c|c|c|c|c|c|c|}
\hline \multirow{2}{*}{$\begin{array}{c}Q_{o}(\% \text { of } \\
\left.Q_{\text {rated }}\right)\end{array}$} & \multirow{2}{*}{$K_{u}$} & \multicolumn{7}{|c|}{$P_{o}\left(\%\right.$ of $\left.P_{\text {rated }}\right) \quad$ Note: $S_{\text {rated }}=1$ p.u. and $p f_{\text {rated }}=0.8$} \\
\hline & & 5 & 10 & 30 & 50 & 70 & 90 & 95 \\
\hline 5 & 0.782 & 0.159 & 0.103 & 0.134 & 0.146 & 0.152 & 0.156 & 0.151 \\
\hline 10 & 0.786 & & 0.161 & 0.115 & 0.131 & 0.141 & 0.148 & 0.149 \\
\hline 30 & 0.800 & & & 0.167 & 0.105 & 0.111 & 0.120 & 0.122 \\
\hline 50 & 0.814 & & & & 0.174 & 0.110 & 0.107 & 0.107 \\
\hline 70 & 0.837 & & & & & 0.181 & 0.116 & 0.112 \\
\hline 90 & 0.844 & & & & & & 0.188 & 0.158 \\
\hline 95 & 0.848 & & & & & & 0.244 & 0.189 \\
\hline
\end{tabular}

A factor $K_{u i}$ is introduced into the expression in order to simplify the relationship between $X_{v i}$ and $Q_{i}$.

$$
K_{u i}=\frac{R_{e i}}{R_{e i}^{d}}=\frac{X_{e i}}{X_{e i}^{d}}=\frac{\left|u_{o i}\right|^{2}}{\left|u_{o d i}^{d}\right|^{2}}
$$

TABLE I. THE CORRESPONDING $\kappa_{i}$ VALUES FOR DIFFERENT OPERATING POINTS WITH $c=0.2$

TABLE II. SYSTEM PARAMETERS

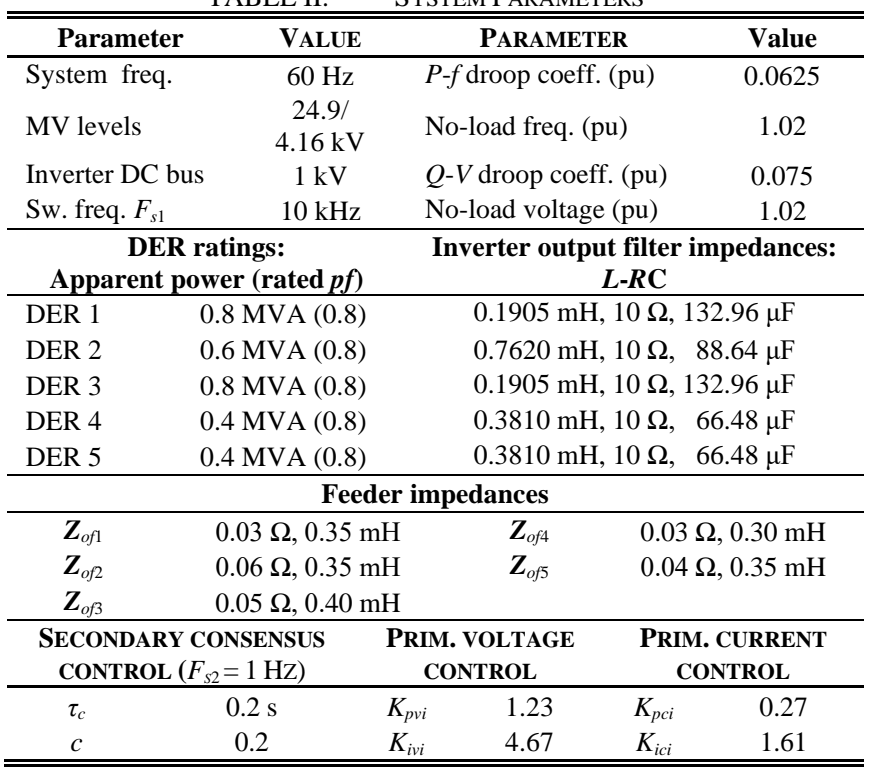

Notice that $K_{u i}$ value remains typically near to one as the virtual voltage drop is typically kept small as compared to the rated voltage value. On this basis, if both $K_{u i}$ and $P_{i}$ do not change significantly during $Q_{i}$ correction (due to $X_{v i}$ adjustment), the dynamical relationship between $X_{v i}$ and $Q_{i}$ can be approximated to a linear, operating-point-dependent relation through timederivative of (24):

$$
\dot{X}_{v i}=F_{i}\left(V_{i}^{*}, n, P_{o i}, Q_{o i}, K_{u i}\right) \cdot \dot{Q}_{i}
$$

where the full expression of $F_{i}$ is given in the appendix. $P_{o i}$ and $Q_{o i}$ are the power values at the steady state operating point about which the linearization is made.

Next, the relationship between $X^{d}{ }_{e i}$ and $Q_{i}$ is to be established. From $X^{d}{ }_{e i}$ expression in (17), and assume that the active power $P_{i}$ does not change significantly during $Q_{i}$ correction, the dynamical relationship between $X_{e i}^{d}$ and $Q_{i}$ can again be approximated to a linear, operating-point-dependent relationship:

$$
\dot{X}_{e i}^{d}=G_{i}\left(V_{i}^{*}, n, P_{o i}, Q_{o i}\right) \cdot \dot{Q}_{i}
$$

where the full expression of $G_{i}$ is given in the appendix. Then, with (26) and (27), the relationship between $X_{v i}$ and $X^{d}{ }_{e i}$ can be given by

$$
\dot{X}_{v i}=H_{i}\left(V_{i}^{*}, n, P_{o i}, Q_{o i}, K_{u i}\right) \cdot \dot{X}_{e i}^{d}
$$

where $H_{i}=F_{i} / G_{i}$. Based on the basic principle of linearization about an operating point, (28) gives a simplified linear relationship between the time-derivative of $X_{v i}$ and timederivative of $X^{d}{ }_{e i}$. By substituting (28) into (18), and rewrite the consensus algorithm, we get: 


$$
\dot{X}_{v i}=-\kappa_{i} \sum_{j \in N_{i}} a_{i j}\left(X_{e i}^{d}-X_{e j}^{d}\right)
$$

where $\kappa_{i}={ }_{c} H_{i}\left(V_{i}^{*}, n, P_{o i}, Q_{o i}, K_{u i}\right)$. Reactive power correction through adaptive VOI is fundamentally a non-linear control problem. This is reflected in the coupling gain $\kappa_{i}$ (29) through its operating-point dependent nature. Upon first-order discretization, the virtual output reactance can be found:

$$
\begin{aligned}
& X_{v i}(k+1)=X_{v i}(k)+T_{s} \Delta X_{v i}(k) \\
& \Delta X_{v i}(k)=-\kappa_{i} \sum_{j \in N_{i}} a_{i j}\left[X_{e i}^{d}(k)-X_{e j}^{d}(k)\right]
\end{aligned}
$$

It is well-established that consensus stability is guaranteed if sufficient condition (2) is met. However, as revealed from (29), the effective local gain $\kappa_{i}$ for a given global coupling gain $c$ is essentially dependent on the local operating point. There are two possible ways of setting the local gain $\kappa_{i}$ : (i) compute the local $H_{i}$ value on the fly and update $\kappa_{i}$ accordingly; (ii) consider the smallest value of $H_{i, \min }$ for a given range of operating points and use $\mathrm{cH}_{i, \min }$ as the global gain $\kappa$ (common to all DERs). This work considers the second way as it is expected to give a more reliable consensus control but with a small penalty on the correction dynamics. The possible values of $\kappa$ for a given range of operating points (i.e. different $P_{o}$ and $Q_{o}$, with a rated power factor of 0.8 ) is summarized in Table I. The calculation assumes that $u_{o i}$ is fixed at 0.9 and the load power factor is always kept equal or above 0.8 (grey-shaded column is below 0.8). Based on the analysis, it is recommended that the coupling gain $\kappa_{i}$ should be chosen at middle-loading (e.g. one with 50\% $P_{\text {rated }}$ and 30\% $Q_{\text {rated }}$ ).

Lastly, communication delay is inevitable in practice. Based on (3), the proposed consensus control with communication delay can be expressed as:

$$
\Delta X_{v i}(k)=-\kappa_{i} \sum_{j \in N_{i}} a_{i j}\left[X_{e i}^{d}(k-\tau)-X_{e j}^{d}(k-\tau)\right]
$$

\section{RESUlTS AND DisCUSSION}

An islanded microgrid network and the corresponding DERs' local controllers are implemented in DIgSILENT PowerFactory while the distributed secondary control is in Python. MatrikonOPC is used as the OPC server to facilitate the information exchange among the DERs. DIgSILENT PowerFactory and Python are the OPC clients and they interface with the OPC server through respective interfaces (ComLink in PowerFactory and OpenOPC in Python). See [35] and reference therein for details.

The proposed secondary reactive power sharing control is verified on an islanded microgrid modified from the IEEE 34 Node Test Feeder [36]. Basic parameters of the DERs and the modified microgrid network are tabulated in Table II. Some of the network features are highlighted below:

(a) DERs with different power ratings are connected to the islanded microgrid via Y-Y, $400 \mathrm{~V} / 24.9 \mathrm{kV}$ (4.16 kV for the short lateral) transformer. Their placement in the network, as shown in Fig. 4, are based on $P V$ curve analysis of PowerFactory [37];

(b) Shunt capacitors from the original IEEE 34 Node Test Feeder are kept in the network (at bus 844 and 848) and they are of a total rating of 0.75 MVar;

(c) $L C$ filters with resistive damping are used as the switching

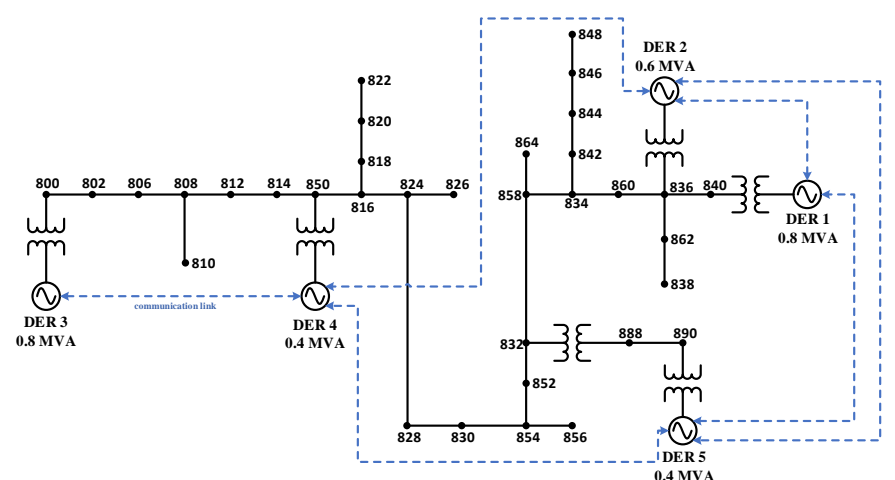

Fig. 4. A large-area islanded microgrid modified from the IEEE 34 Node Test Feeder (with actual network impedances).
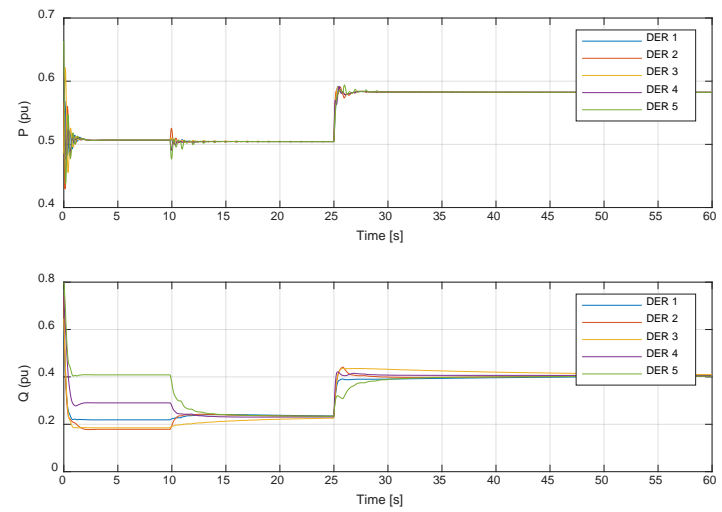

Fig. 5. Default steady state and load up-stepping with fixed $\kappa$ : active and reactive output power of DERs for the standard droop control (before $10 \mathrm{~s}$ ) without correction (after $10 \mathrm{~s}$ ) with correction and (at $25 \mathrm{~s}$ ) load step change.

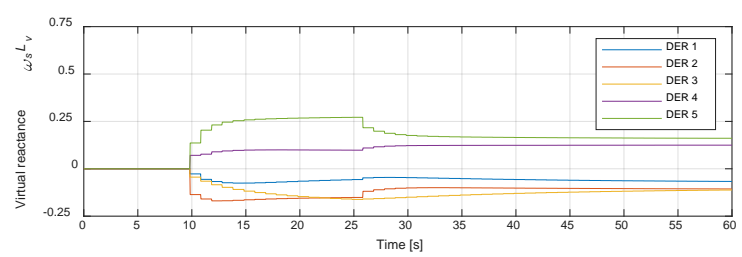

Fig. 6. Default steady state and load up-stepping with fixed $\kappa$ : adaptive virtual reactance generated.

harmonic filters,

(d) The Laplacian matrix of the communication graph is

$$
\boldsymbol{L}_{G}=\left[\begin{array}{ccccc}
2 & -1 & 0 & 0 & -1 \\
-1 & 3 & 0 & -1 & -1 \\
0 & 0 & 1 & -1 & 0 \\
0 & -1 & -1 & 3 & -1 \\
-1 & -1 & 0 & -1 & 3
\end{array}\right]
$$

Fig. 4 depicts the single-line diagram of the large-area microgrid modified from IEEE 34 Node Test Feeder. Three case studies have been conducted: default steady state and load change; DER plug and play; and communication link delay.

\section{A. Case Study 1: Default Steady State and Load Change with Coupling Gain Consideration}

A total load of 1.52 MW and 1.27 MVar passive load are present in the islanded microgrid network. Before $t=10 \mathrm{~s}$, the DERs are initially controlled through the standard droop scheme without any reactive power sharing correction. It can be 

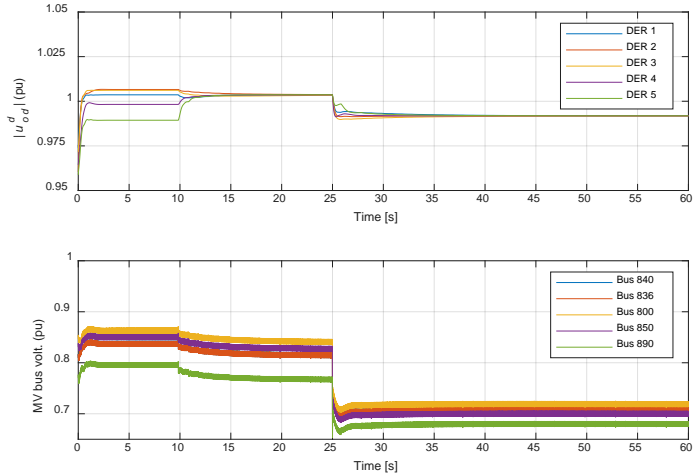

Fig. 7. Default steady state and load up-stepping with fixed $\kappa$ : the corresponding droop output voltage references and voltage magnitudes at respective MV buses.

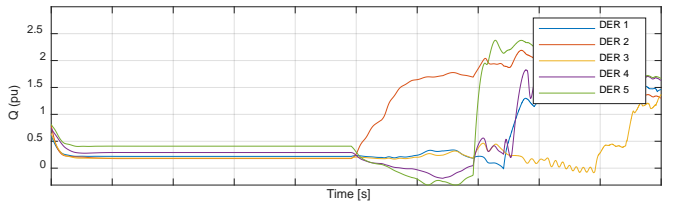

Fig. 8. Default steady state with fixed $\kappa=0.3$ : DERs' reactive output power.
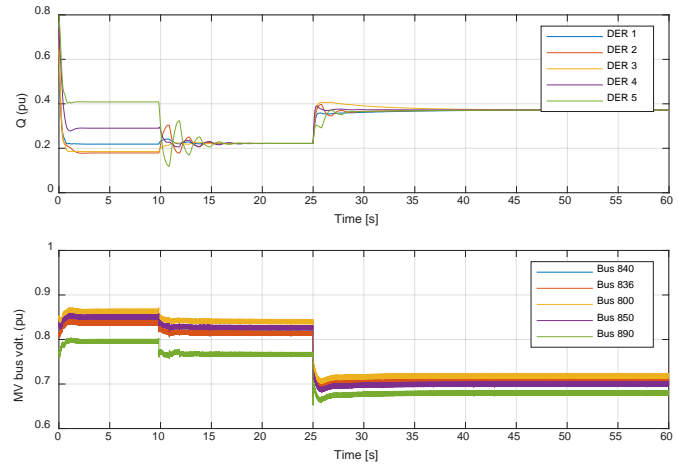

Fig. 9. Default steady state and load up-stepping with adaptively tuned $\kappa$ : DERs' reactive output power and voltage magnitudes at respective MV buses.

established from Fig. 5 that the active power is always proportionally shared, but the reactive power is not. At $t=10 \mathrm{~s}$, the proposed reactive power sharing control scheme is activated. Based on the established tuning guideline, $\kappa$ is globally tuned to 0.105 . It is seen clearly that the load reactive power is now proportionally shared among the DERs. The corresponding virtual reactance profiles in Fig. 6 show that the virtual reactance can take both positive and negative values.

Traces in Fig. 7 show that upon steady state the droop voltage references also equalize but the MV bus voltages have been lowered further. Subsequently, an additional load is connected to bus 814 at $t=25 \mathrm{~s}$ during which the total load demand of the islanded microgrid is increased to $1.75+j 1.66$ MVA. Figs. 5-7 show that the proposed control scheme responds accordingly without noticeable large transients.

Fig. 8 shows that control instability occurs when $\kappa$ is globally tuned to 0.3 despite that $\kappa$ is less than $1 / d_{\max }$ (as in (2)). This is due to the fact that the dynamical relationship between $X_{v i}$ and $X^{d}{ }_{e i}$ has not been accounted for in the consensus protocol (30), the consensus theorem, i.e. (2), therefore cannot be applied directly. On the other hand, the performance of an adaptively tuned control scheme (i.e with local $\kappa_{i}$ gain defined by the local operating points of ${ }^{d} d i, K_{u i}, P_{o i}$, and $Q_{o i}$ ) is shown in Fig. 9. A
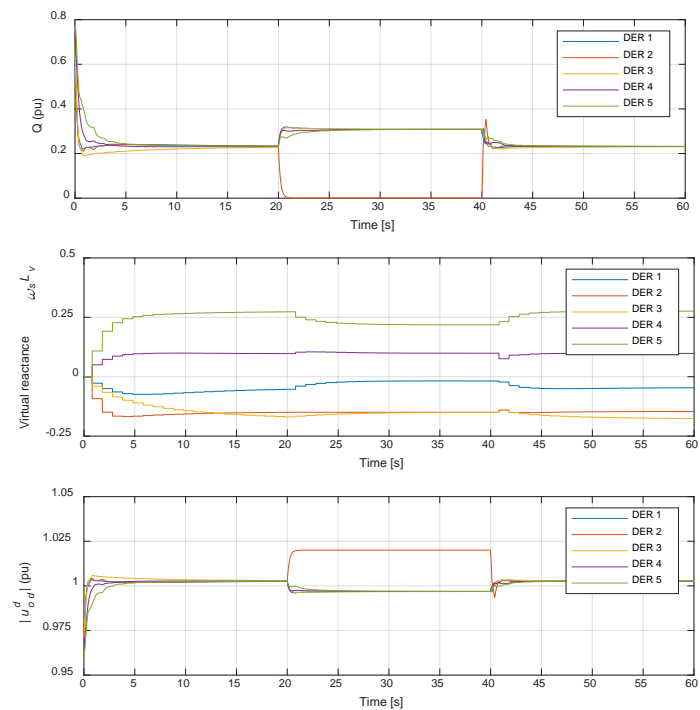

Fig. 10. DER 2 plug-and-play capability: DERs' reactive output power, adaptive virtual reactance generated and the corresponded droop output voltage references.
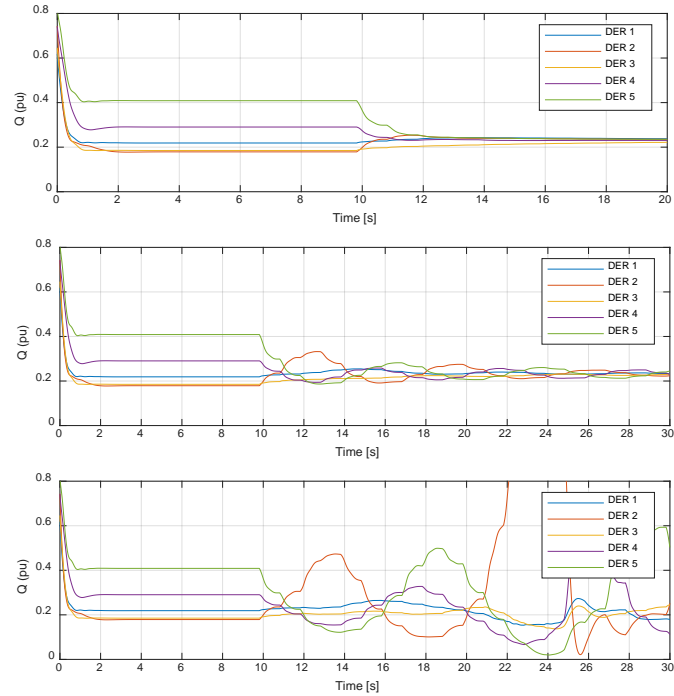

Fig. 11. DERs' reactive output power with communication link time delay of (top) $0.6 \mathrm{~s} \mathrm{(mid)} 1.4 \mathrm{~s}$ and (bottom) $1.8 \mathrm{~s}$.

stable, underdamped performance is noted. This finding agrees with the theoretical expectation in Section IV. However, as mentioned, in order to ensure a stable consensus control, it is preferable to tune gain $\kappa$ globally to the mid-loading value (i.e. being 0.105 in Table I). This is done for subsequent studies.

\section{B. Case Study 2: Plug-and-Play Capability}

The dynamic performance of the proposed VOI control scheme considering the plug-and-play capability is studied here. Notice that a DER's disconnection means that all communication links between the DER and the remaining DERs are lost. In order to ensure a stable consensus system, it is assumed that the communication links among the remaining DERs still maintain a connected graph.

The islanded microgrid operates under default loading condition with the proposed control scheme activated from $t=0$ $\mathrm{s}$. At $t=20 \mathrm{~s}$, DER 2's circuit breaker is opened, and it is reclosed at $t=40 \mathrm{~s}$. As seen in Fig. 10, proportional reactive power sharing is retained by the remaining DERs. It can be noticed that the reactive powers of the remaining DERs increase 
and their corresponding droop voltage reference decreases. With the reconnection of DER 2 at $t=40 \mathrm{~s}$, the reactive load power is almost instantaneously re-distributed among the DERs with some small overshoots.

\section{Case Study 3: Effect of Communication Link Delays}

The robustness of the distributed control scheme against communication link delays is examined next. Based on the consensus theorem (3-4) and (31), the maximum allowable time delay is $\tau_{\max }=1.75 \mathrm{~s}$ (i.e. with the maximum eigenvalue of $\lambda_{\max }$ $=4.48$ and the coupling gain $c=0.2$ ). In this case study, the time delay $\tau$ is set to be $0.6,1.4$ and $1.8 \mathrm{~s}$, respectively. Fig. 11 shows the corresponding reactive output powers of the DERs with the proposed control scheme activated at $t=10 \mathrm{~s}$. It can be seen that proportional reactive power sharing is achieved for the case of $\tau=0.6 \mathrm{~s}\left(<<\tau_{\max }\right)$. For the case of $\tau=1.4 \mathrm{~s}$, power oscillation can be seen before the final steady state. However, for the case of $\tau=1.8 \mathrm{~s}\left(>\tau_{\max }\right)$, the islanded microgrid is unable to maintain a stable operation. This essentially verifies the communication delay discussion described in Sections II and III. It is worth highlighting here that the compliance is only possible if the control gain $\kappa$ is tuned by the proposed tuning guideline; if one were not to consider the operating-pointdependent $H$ or were to append proportional-integral controllers subsequent to the consensus control, (4) will not be applicable.

\section{CONCLUSION}

In this paper, we propose a novel droop-equivalentreactance-based consensus adaptive VOI control to realize accurate reactive power sharing among DERs in a radial microgrid. The control scheme only needs a sparse communication structure and eliminates the needs for a central control. The proposed control scheme does not rely on the local proportional-integral controllers and therefore, there is effectively one coupling gain to be tuned. A systematic tuning guideline with linearization about practical operating points is established and proven. The proposed control scheme has been tested for different test scenarios: steady state, transients (load up-stepping), plug-and-play, and communication delay, and the results successfully justify the claims.

\section{APPENDIX}

$$
\begin{aligned}
F_{i}= & -\frac{9}{\sqrt{\frac{9 K_{u i}\left(V_{i}^{*}-n Q_{o i}\right)^{4}}{P_{o i}^{2}+Q_{o i}^{2}}-\frac{9 K_{u i}^{2} P_{o i}^{2}\left(V_{i}^{*}-n Q_{o i}\right)^{4}}{\left(P_{o i}^{2}+Q_{o i}^{2}\right)^{2}}}}\left[\frac{K_{u i} Q_{o i}\left(V_{i}^{*}-n Q_{o i}\right)^{4}}{\left(P_{o i}^{2}+Q_{o i}^{2}\right)^{2}}\right. \\
& -\frac{2 K_{u i}^{2} P_{o i}^{2} Q_{o i}\left(V_{i}^{*}-n Q_{o i}\right)^{4}}{\left(P_{o i}^{2}+Q_{o i}^{2}\right)^{3}}+\frac{2 K_{u i}\left(V_{i}^{* 2} n-3 V_{i}^{* 2} n^{2} Q_{o i}+3 V_{i}^{*} n^{3} Q_{o i}{ }^{2}-n^{4} Q_{o i}^{3}\right)}{P_{o i}+Q_{o i}^{2}} \\
& \left.-\frac{2 K_{u i}^{2} P_{o i}^{2}\left(V_{i}^{* 2} n-3 V_{i}^{* 2} n^{2} Q_{o i}+3 V_{i}^{*} n^{3} Q_{o i}{ }^{2}-n^{4} Q_{o i}^{3}\right)}{\left(P_{o i}^{2}+Q_{o i}^{2}\right)^{2}}\right]+\frac{6 K_{u i} Q_{o i}^{2}\left(V_{i}^{*}-n Q_{o i}\right)^{2}}{\left(P_{o i}^{2}+Q_{o i}^{2}\right)^{2}} \\
& -\frac{3 K_{u i}\left(V_{i}^{* 2}-4 V_{i}^{*} n Q_{o i}+3 n^{2} Q_{o i}^{2}\right)}{P_{o i}^{2}+Q_{o i}^{2}} \\
& G_{i}=\frac{3 V_{i}^{* 2} P_{o i}^{2}-3 V_{i}^{* 2} Q_{o i}^{2}-12 V_{i}^{*} n P_{o i}{ }^{2} Q_{o i}+9 n^{2} P_{o i}^{2} Q_{o i}^{2}+3 n^{2} Q_{o i}^{4}}{\left(P_{o i}^{2}+Q_{o i}^{2}\right)^{2}}
\end{aligned}
$$

\section{REFERENCES}

[1] Y. Han, H. Li, P. Shen, E. A. A. Coelho, and J. M. Guerrero, "Review of active and reactive power sharing strategies in hierarchical controlled microgrids," IEEE Trans. Power Electron., vol. 32, no. 3, pp. 2427-2451, Mar. 2017.

[2] Y. Han, P. Shen, X. Zhao, and J. M. Guerrero, "An enhanced power sharing scheme for voltage unbalance and harmonics compensation in an islanded ac microgrid," IEEE Trans. Energy Convers., vol. 31, no. 3, pp. 1037-1050, Sep. 2016.

[3] Y. W. Li and C. N. Kao, "An accurate power control strategy for powerelectronics-interfaced distributed generation units operating in a lowvoltage multibus microgrid,” IEEE Trans. Power Electron., vol. 24, no. 12, pp. 2977-2988, Dec. 2009.

[4] T. Qunais and M. Karimi-Ghartemani, "Systematic modeling of a class of microgrids and its application to impact analysis of cross-coupling droop terms," IEEE Trans. Energy Convers., vol. 34, no. 3, pp. 1632-1643, Sep. 2019.

[5] Q. Shafiee, J. M. Guerrero, and J. C. Vasquez, "Distributed secondary control for islanded microgrids-a novel approach,” IEEE Trans. Power Electron., vol. 29, no. 2, pp. 1018-1031, Feb. 2014.

[6] H. Mahmood, D. Michaelson, and J. Jiang, "Reactive power sharing in islanded microgrids using adaptive voltage droop control,” IEEE Trans. Smart Grid, vol. 6, no. 6, pp. 3052-3060, Nov. 2015.

[7] X. Yang, Y. Du, J. Su, L. Chang, Y. Shi, and J. Lai, "An optimal secondary voltage control strategy for an islanded multibus microgrid," IEEE J. Emerg. Sel. Top. Power Electron., vol. 4, no. 4, pp. 1236-1246, Dec. 2016.

[8] A. Bidram, A. Davoudi, and F. L. Lewis, "A multiobjective distributed control framework for islanded AC microgrids," IEEE Trans. Ind. Informatics, vol. 10, no. 3, pp. 1785-1798, Aug. 2014.

[9] J. W. Simpson-Porco, Q. Shafiee, F. Dorfler, J. C. Vasquez, J. M. Guerrero, and F. Bullo, "Secondary frequency and voltage control of islanded microgrids via distributed averaging," IEEE Trans. Ind. Electron., vol. 62, no. 11, pp. 7025-7038, Nov. 2015.

[10] R. Han, L. Meng, G. Ferrari-Trecate, E. A. A. Coelho, J. C. Vasquez, and J. M. Guerrero, "Containment and consensus-based distributed coordination control to achieve bounded voltage and precise reactive power sharing in islanded AC microgrids," IEEE Trans. Ind. Appl., vol. 53, no. 6, pp. 5187-5199, Dec. 2017.

[11] M. A. Mahmud, M. J. Hossain, H. R. Pota, and A. M. T. Oo, "Robust nonlinear distributed controller design for active and reactive power sharing in islanded microgrids," IEEE Trans. Energy Convers., vol. 29, no. 4, pp. 893-903, Dec. 2014.

[12] M. S. Golsorkhi, Q. Shafiee, D. D. C. Lu, and J. M. Guerrero, "Distributed control of low-voltage resistive ac microgrids," IEEE Trans. Energy Convers., vol. 34, no. 2, pp. 573-584, Jun. 2019.

[13] J. Schiffer, T. Seel, J. Raisch, and T. Sezi, "Voltage stability and reactive power sharing in inverter-based microgrids with consensus-based distributed voltage control,” IEEE Trans. Control Syst. Technol., vol. 24, no. 1, pp. 96-109, Jan. 2016.

[14] J. M. Guerrero, L. Gracia de Vicuna, J. Matas, M. Castilla, and J. Miret, "Output impedance design of parallel-connected UPS inverters with wireless load-sharing control," IEEE Trans. Ind. Electron., vol. 52, no. 4, pp. 1126-1135, Aug. 2005.

[15] J. M. Guerrero, J. Matas, L. Gracia de Vicuna, M. Castilla, and J. Miret, "Decentralized control for parallel operation of distributed generation inverters using resistive output impedance," IEEE Trans. Ind. Electron., vol. 54, no. 2, pp. 994-1004, Apr. 2007.

[16] J. He, Y. W. Li, J. M. Guerrero, F. Blaabjerg, and J. C. Vasquez, “An islanding microgrid power sharing approach using enhanced virtual impedance control scheme," IEEE Trans. Power Electron., vol. 28, no. 11, pp. 5272-5282, Nov. 2013.

[17] Y. Zhu, F. Zhuo, F. Wang, B. Liu, R. Gou, and Y. Zhao, “A virtual impedance optimization method for reactive power sharing in networked microgrid,” IEEE Trans. Power Electron., vol. 31, no. 4, pp. 2890-2904, Apr. 2016.

[18] Z. Peng, J. Wang, D. Bi, Y. Wen, Y. Dai, X. Yin, and Z. J. Shen, "Droop control strategy incorporating coupling compensation and virtual impedance for microgrid application," IEEE Trans. Energy Convers., vol. 34, no. 1, pp. 277-291, Mar. 2019.

[19] M. Savaghebi, A. Jalilian, J. C. Vasquez, and J. M. Guerrero, "Autonomous voltage unbalance compensation in an islanded droopcontrolled microgrid," IEEE Trans. Ind. Electron., vol. 60, no. 4, pp. 1390-1402, Apr. 2013. 
[20] M. Savaghebi, A. Jalilian, J. C. Vasquez, and J. M. Guerrero, "Secondary control scheme for voltage unbalance compensation in an islanded droopcontrolled microgrid," IEEE Trans. Smart Grid, vol. 3, no. 2, pp. 797807, Jun. 2012

[21] J. He and Y. W. Li, "Analysis, design, and implementation of virtual impedance for power electronics interfaced distributed generation," IEEE Trans. Ind. Appl., vol. 47, no. 6, pp. 2525-2538, Dec. 2011.

[22] Y. Zhu, B. Liu, F. Wang, F. Zhuo, and Y. Zhao, "A virtual resistance based reactive power sharing strategy for networked microgrid," in Int. Conf. on Power Electronics and ECCE Asia, 2015, pp. 1564-1572.

[23] T. V. Hoang and H. H. Lee, "An adaptive virtual impedance control scheme to eliminate the reactive-power-sharing errors in an islanding meshed microgrid,” IEEE J. Emerg. Sel. Top. Power Electron., vol. 6, no. 2, pp. 966-976, Jun. 2018.

[24] H. Zhang, S. Kim, Q. Sun, and J. Zhou, "Distributed adaptive virtual impedance control for accurate reactive power sharing based on consensus control in microgrids,” IEEE Trans. Smart Grid, vol. 8, no. 4, pp. 1749-1761, Jul. 2017.

[25] H. Mahmood, D. Michaelson, and J. Jiang, "Accurate reactive power sharing in an islanded microgrid using adaptive virtual impedances," IEEE Trans. Power Electron., vol. 30, no. 3, pp. 1605-1617, Mar. 2015.

[26] B. Liu, Z. Liu, J. Liu, R. An, and S. Song, "A novel microgrid power sharing scheme enhanced by a non-intrusive feeder impedance estimation method," in Int. Power Electron. Conf., 2018, pp. 3924-3928.

[27] D. K. Dheer, O. V. Kulkarni, S. Doolla, and A. K. Rathore, "Effect of reconfiguration and meshed networks on the small-signal stability margin of droop-based islanded microgrids," IEEE Trans. Ind. Appl., vol. 54, no. 3, pp. 2821-2833, May 2018.

[28] A. Stanoev and D. Smilkov, "Consensus theory in networked systems," in Consensus and Synchronization in Complex Networks, L. Kocarev, Ed. Berlin: Springer-Verlag Berlin, 2013, pp. 1-22.

[29] R. Olfati-Saber and R. M. Murray, "Consensus problems in networks of agents with switching topology and time-delays," IEEE Trans. Automat. Contr., vol. 49, no. 9, pp. 1520-1533, Sep. 2004.

[30] R. Olfati-Saber, J. A. Fax, and R. M. Murray, "Consensus and cooperation in networked multi-agent systems," Proc. IEEE, vol. 95, no. 1, pp. 215233, Jan. 2007.

[31] J. Zhou, S. Kim, H. Zhang, Q. Sun, and R. Han, "Consensus-based distributed control for accurate reactive, harmonic, and imbalance power sharing in microgrids,” IEEE Trans. Smart Grid, vol. 9, no. 4, pp. 24532467, Jul. 2018.

[32] J. M. Rey, C. X. Rosero, M. Velasco, P. Marti, J. Miret, and M. Castilla, "Local frequency restoration for droop-controlled parallel inverters in islanded microgrids," IEEE Trans. Energy Convers., vol. 34, no. 3, pp. 1232-1241, Sep. 2018.

[33] N. Pogaku, M. Prodanović, and T. C. Green, "Modeling, analysis and testing of autonomous operation of an inverter-based microgrid," IEEE Trans. Power Electron., vol. 22, no. 2, pp. 613-625, Mar. 2007.

[34] Y. Zhu, Q. Fan, B. Liu, and T. Wang, "An enhanced virtual impedance optimization method for reactive power sharing in microgrids," IEEE Trans. Power Electron., vol. 33, no. 12, pp. 10390-10402, Sep. 2018.

[35] Y. C. C. Wong, C. S. Lim, M. D. Rotaru, A. Cruden, and K. Xin, "Reactive power sharing study of an islanded microgrid in DIgSILENT PowerFactory," in Int. Conf. on Renewable Energy Res. and Appl., 2018, pp. 1-6.

[36] IEEE Power and Energy Society, "IEEE 34 node test feeder," 2010. [Online]. Available: http://sites.ieee.org/pes-testfeeders/resources/.

[37] Q. Fu, L. F. Montoya, A. Solanki, A. Nasiri, V. Bhavaraju, T. Abdallah, and D. C. Yu, "Microgrid generation capacity design with renewables and energy storage addressing power quality and surety," IEEE Trans. Smart Grid, vol. 3, no. 4, pp. 2019-2027, Sep. 2012.

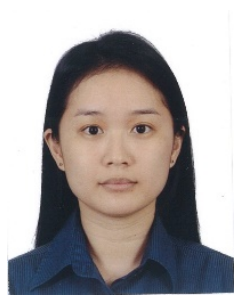

Yi Chyn Cassandra Wong (S'18) received the B.Eng. (Hons.) degree in electrical power engineering from the Curtin University, Malaysia, in 2016. She is currently working toward the split-site Ph.D. degree with the University of Southampton, U.K., and the University of Southampton Malaysia.

She was a research intern with the Experimental Power Grid Centre, Agency for Science, Technology, and Research (A*STAR), Singapore, from August to October 2017. Her research interests include power quality improvement, optimization control, and distributed cooperative control in microgrid.

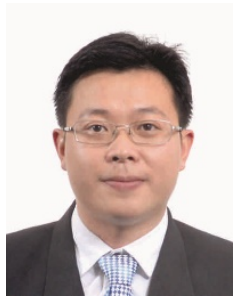

Chee Shen Lim (SM'19) received the B.Eng. degree (Hons.) in electrical engineering from the University of Malaya, Malaysia, in 2009, and the joint-university Ph.D. degrees in power electronics and drives from the University of Malaya and Liverpool John Moores University, U.K., in 2013.

From 2009 to 2013, he was a Research Assistant with the Power Energy Dedicated Advanced Centre, University of Malaya. From 2013 to 2015, he was a Research Scientist with the Experimental Power Grid Centre, A*STAR, Singapore. He is currently an Assistant Professor of electrical and electronic engineering with the University of Southampton Malaysia. His research interests include advanced model predictive control design, multiphase motor drives, gridconnected converter control, and microgrid hierarchical control. He also serves as an Associate Editor for the IET Electric Power Applications.

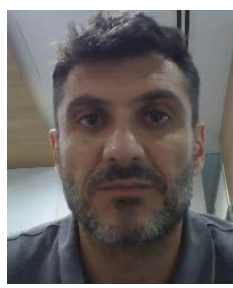

Mihai Dragos Rotaru (M’00) received the B.Eng. and M.Sc. degrees in electrical engineering from the Technical University Cluj Napoca, Romania, in 1996 and 1997, respectively, and the Ph.D. degree in Electrical Engineering from the University of Southampton, U.K., in 2001.

He was an assistant professor then an associate professor with the School of Electronics and Computer Science of University of Southampton from 2007 to 2019. He also served as the Electrical and Electronic Engineering program leader at the University of Southampton Malaysia from 2013 to 2019. He is currently a Senior Scientist with the Institute of Microelectronics, A*STAR, Singapore. His research interests and expertise include simulation and modelling of complex applied electromagnetic problems.

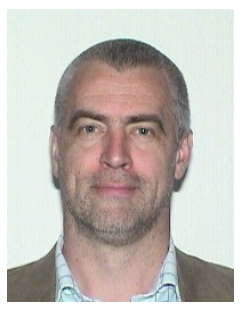

Andrew Cruden received the B.Eng., M.Sc., and Ph.D. degrees from the University of Strathclyde, Glasgow, U.K., in 1989, 1990, and 1998, respectively, all in electronic and electrical engineering. He is currently the Head of the Energy Technologies Research Group and a Professor of Energy Technology with the University of Southampton, U.K.

He has significant experience in the field of energy storage and electric vehicles, covering Vehicle-to-Grid (V2G), new battery technologies (e.g. Aluminium-ion cells), and flow cells (e.g. soluble lead flow battery). He has previously worked with fuel cell technology and condition monitoring of wind turbines. He is a member of the Training and Diversity Panel of the UK's Faraday Institution and Co-Director of the EPSRC Centre for Doctoral Training (CDT) in Energy Storage and its Applications.

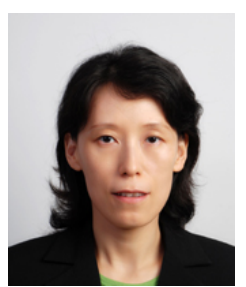

Kong Xin received the B.Eng. and M.Eng. degrees in electrical engineering from Xi'an Jiaotong University, China, in 1994 and 1997, respectively, and the Ph.D. degree in electrical engineering from the National University of Singapore, Singapore, in 2009.

She currently serves as a Senior Research Scientist and the Deputy Program Director in Energy Research Institute at ERI@N, Nanyang Technological University, Singapore. Her research interests include modern power system modelling and analysis, renewable energy integration, distributed microgrid control, and power electronic design and control for grid application. 